\title{
Telmisartan inhibits bladder smooth muscle fibrosis in neurogenic bladder rats
}

\author{
QIAN LIU, RUOYI WANG, NAN MA, CHUNTIAN WANG and WEIXIU CHEN \\ Department of Pediatric Surgery, The Second Hospital of Shandong University, Jinan, Shandong 250033, P.R. China
}

Received October 20, 2021; Accepted December 7, 2021

DOI: $10.3892 / \mathrm{etm} .2022 .11140$

\begin{abstract}
Hypertension is associated with bladder symptoms. The present study investigated whether an angiotensin receptor blocker could improve the symptoms and pathological changes associated with a neurogenic bladder (NB). A Sprague-Dawley rat model of NB was constructed. Rats in the sham and model groups were gavaged with saline, and rats in the treatment group were gavaged with telmisartan. Urodynamic parameters, including maximum cystometric capacity, residual urine volume, bladder wet weight, bladder compliance and detrusor pressure, were detected. Masson and $\mathrm{H} \& \mathrm{E}$ staining were performed to assess bladder fibrosis and histopathological changes. The expression levels of basic fibroblast growth factor (bFGF), TGF- $\beta 1$, Collagen I, Collagen III, and $\alpha$-smooth muscle actin ( $\alpha$-SMA) were also measured by reverse transcription-quantitative PCR, western blotting and immunohistochemistry. The model rats exhibited symptoms and pathological changes associated with NB. Treatment with telmisartan reduced maximum cystometric capacity, residual urine volume, bladder compliance and bladder wet weight, and increased detrusor pressure in model rats. The tissue staining results showed that telmisartan exerted an antifibrotic effect. In addition, telmisartan inhibited the expression of bFGF, TGF- $\beta 1$, Collagen I, Collagen III and $\alpha$-SMA in model rats. Therefore, the results of the present study indicated that telmisartan may serve as a potential therapeutic agent for NB.
\end{abstract}

\section{Introduction}

A spinal cord injury (SCI) can lead to the deterioration of various physiological functions, especially bladder function (1). After an SCI, nerve impulses from the bladder and sphincter do not

Correspondence to: Dr Weixiu Chen, Department of Pediatric Surgery, The Second Hospital of Shandong University, 247 Beiyuan Street, Jinan, Shandong 250033, P.R. China

E-mail: chenweixiu@126.com

Key words: neurogenic bladder, telmisartan, bladder compliance, basic fibroblast growth factor, TGF- $\beta$, collagen, $\alpha$-smooth muscle actin reach the brain, and cannot be felt by the affected individual. This type of bladder dysfunction is referred to a neurogenic bladder (NB) $(2,3)$. A significant pathological feature of NB is detrusor fibrosis, which occurs when a large number of collagen fibers are deposited between the muscle bundles (4). This collage deposition leads to detrusor contracture, detrusor weak contraction, bladder storage and emptying disorders, upper urinary tract damage and serious complications, such as renal failure $(5,6)$.

The treatment strategies for SCI-induced NB include psychotherapy, electrical stimulation, chemotherapy, intermittent catheterization and surgery $(7,8)$. Bladder fibrosis has been reported to occur over time in patients with NB (9), suggesting that fibrosis is an important factor affecting the outcome of therapy. Studies have shown that both TGF- $\beta 1$ and basic fibroblast growth factor (bFGF) are important for the differentiation and proliferation of smooth muscle cells, and activation of these signaling pathways contributes to fibrosis and sclerosis of the bladder wall after an SCI (10-12). Some researchers have found that microRNA-101b downregulates the TGF- $\beta$ signaling pathway by inhibiting TGF- $\beta$ receptor 1 , thereby inhibiting fibrosis. These results indicate that TGF- $\beta 1$ is involved in the fibrosis of bladder tissue. An in vitro study showed that bFGF upregulates the expression of Collagen I (13). However, the mechanism underlying NB fibrosis is not fully understood.

Current treatment strategies for bladder fibrosis include the use of antifibrotic drugs, such as relaxin (14), and the application of stem cell and gene therapy $(15,16)$; however, none of these treatments have been effective. Therefore, developing additional therapies for NB is important. Telmisartan is a new type of antihypertensive drug and a specific angiotensin II receptor antagonist (17). A recent study has shown that angiotensin II can promote cardiac fibrosis by binding to Ang II type I receptors and further promoting the synthesis of TGF- $\beta 1$ (18). However, no clinical guidelines have recommended the use of telmisartan for the treatment of NB. In the present study, the L6-S1 spinal nerves of rats were bilaterally dissected to construct an NB rat model to observe the effects of telmisartan on maximum cystometric capacity, residual urine volume, bladder wet weight, bladder compliance, detrusor pressure and fibrosis-related gene expression. The results of the present study may provide rationale for using telmisartan during the early treatment of NB. 


\section{Materials and methods}

Experimental animals and groupings. To rule out potential confounding effects, only 30 adult male Sprague-Dawley rats (weight, 260 $\pm 10 \mathrm{~g}$; age, 13 weeks) were obtained from Shanghai Sippr-BK Laboratory Animal Co. Ltd. The rats were housed together in a room maintained at $40-60 \%$ relative humidity and $23 \pm 2^{\circ} \mathrm{C}$ with 12 -h light/dark cycles and ad libitum access to food and water. The rats were randomly assigned to the following five groups ( $n=6$ per group): i) Sham treatment (epidural exposure only); ii) spinal cord transection treatment; iii) combined treatment with $0.5 \mathrm{mg} / \mathrm{kg} / \mathrm{day}$ telmisartan (cat. no. S1738; Selleck Chemicals) administered by gavage for 14 consecutive days, plus spinal cord transection; iv) combined treatment with $3 \mathrm{mg} / \mathrm{kg} / \mathrm{day}$ telmisartan administered by gavage for 14 consecutive days, plus spinal cord transection; and v) combined treatment with $6 \mathrm{mg} / \mathrm{kg} / \mathrm{day}$ telmisartan administered by gavage for 14 consecutive days, plus spinal cord transection. The sample size was determined using GPower software (version 3.1.9, University of Dusseldorf, Dusseldorf, Germany). The health and behavior of the animals were monitored every 2 days. After cystometric analysis at the 3 rd week or if a humane endpoint, including listlessness or cessation of eating or drinking cessation, was reached, the rats were euthanized by the intraperitoneal injection of sodium pentobarbital ( $200 \mathrm{mg} / \mathrm{kg}$ body weight) and decapitation. All experimental protocols were approved by the Institutional Animal Care and Use Committee of Shandong University Hospital [approval no. KYLL-2021(LW)013]. All procedures were performed in compliance with guidelines issued by the National Institutes of Health.

NB model preparation. After 1 week of adaptation, the rats were anesthetized and fixed on an operating table in the prone position. A $2 \mathrm{~cm}$ median incision was made in the back skin covering the L6-S1 region. The lamina at the L6-S1 level was removed and the spinal cord was completely severed. In the Sham group, the spinal cord was only exposed and not transected. A gelatin sponge (Ethicon, Inc.) was placed between the severed ends to stop bleeding and prevent healing. Next, the muscle layer and skin layer were separately sutured. After surgery, ampicillin sodium (100 mg/kg; cat. no. S3170; Selleck Chemicals) was intramuscularly injected once a day for 5 consecutive days.

Cystometric analysis. For evaluation of bladder function by cystometry, the rats were anesthetized by inhalation of 1.5-2.0\% isoflurane (cat. no. R510-22; RWD Life Science Inc.) for maintenance and induction at 14 days after the operation. A midline abdominal incision was made and a catheter was inserted via the bladder dome. The catheter was connected to a dual-channel syringe pump (cat. no. HK-400A; Shenzhen Hawk Medical Instrument Co., Ltd.) and a pressure transducer in a urodynamic measurement system (Dantec Menuet). Cystometry was performed as described in a previous study (19). The following parameters were measured: Maximum cystometric capacity, residual urine volume, bladder wet weight, bladder compliance and detrusor pressure.

Masson and $H \& E$ staining. The rat bladder was cut from the bladder neck, and the connective tissue surrounding the bladder wall was removed. Next, the bladder tissues were fixed with 4\% paraformaldehyde (cat. no. P0099; Beyotime Institute of Biotechnology) at room temperature (RT) for $24 \mathrm{~h}$, embedded in paraffin and sectioned into 3-mm thick sections. For Masson staining, the paraffin-embedded sections were deparaffinized with an alcohol gradient and xylene, and then washed with distilled water. The sections were then stained with hematoxylin (cat. no. S2384; Selleck Chemicals) at RT for $5 \mathrm{~min}$, washed with tap water, immersed in a $1 \%$ hydrochloric acid alcohol solution for several seconds and rinsed with running water for several minutes. Next, the sections were stained with ponceau red dye (cat. no. S4497; Selleck Chemicals) at RT for 5-10 min; after which, the tissues were rinsed with distilled water and an aqueous phosphomolybdic acid (Electron Microscopy Sciences) solution for 3-5 min. The sections were then treated with aniline blue solution (cat. no. A9540; Beijing Solarbio Science \& Technology Co., Ltd.) at RT for 5 min, followed by treatment with $1 \%$ glacial acetic acid (Guangzhou Jinhuada Chemical Reagent Co., Ltd.) for $1 \mathrm{~min}$. Finally, the sections were dehydrated using an alcohol gradient and xylene, and then sealed with neutral gum (cat. no. N116470-100 g, Shanghai Aladdin Biochemical Technology Co., Ltd.). Blue collagen fibers, red muscle fibers, red cellulose and red blood cells were observed under a microscope (Olympus Corporation). For H\&E staining, the slides were immersed in hematoxylin at RT for $30 \mathrm{sec}$, rinsed with running water until transparent, stained with eosin at RT for $30 \mathrm{sec}$ and then rinsed again with water. The slides were then air-dried at RT. Subsequently, the slides were sequentially immersed twice in $95 \%$ ethanol solution, twice in $100 \%$ ethanol, twice in a solution of $50 \%$ ethanol and $50 \%$ xylene and twice in $100 \%$ xylene. The slides were then observed under a light microscope (Olympus Corporation).

Reverse transcription-quantitative PCR (RT-qPCR). Total RNA was extracted using TRIzol ${ }^{\circledR}$ reagent (cat. no. 15596018; Thermo Scientific, Inc.) according to the manufacturer's instructions. The amount of RNA was quantified by spectrophotometry. Subsequently, reverse transcription was performed using the Bestar ${ }^{\mathrm{TM}}$ qPCR RT kit according to the manufacturer's protocol (cat. no. 2220; DBI Bioscience), followed by qPCR that was performed by using Bestar ${ }^{\mathrm{TM}}$ qPCR MasterMix (cat. no. 2043; DBI Bioscience, Shanghai, China) on an Mx3000P qPCR instrument (Stratagene; Agilent Technologies, Inc.). The following thermocycling conditions were used for qPCR: Melting at $95^{\circ} \mathrm{C}$ for $2 \mathrm{~min}$; followed by 40 cycles of denaturation at $94^{\circ} \mathrm{C}$ for $20 \mathrm{sec}$, annealing at $58^{\circ} \mathrm{C}$ for $20 \mathrm{sec}$, elongation at $72^{\circ} \mathrm{C}$ for $20 \mathrm{sec}$ and $72^{\circ} \mathrm{C}$ complete elongation for $5 \mathrm{~min}$. The primers used for qPCR were synthesized by Sangon Biotech Co., Ltd. and are listed in Table I. mRNA expression levels were quantified using the $2^{-\triangle \Delta C q}$ method (20) and normalized to the internal reference gene GAPDH.

Western blotting. Freshly harvested bladders were homogenized in RIPA lysis buffer (cat. no. P0013C; Beyotime Institute of Biotechnology) and the amount of soluble protein in each homogenate was quantified using a BCA Protein Assay kit (cat. no. 23225; Thermo Fisher Scientific, Inc.). Subsequently, soluble protein $(30 \mu \mathrm{g})$ was separated by $10 \%$ SDS-PAGE and 
Table I. Sequences of primers used for reverse transcriptionquantitative PCR.

\begin{tabular}{ll} 
Gene & \multicolumn{1}{c}{ Sequence (5'-3') } \\
\hline H-GAPDH & F: TGTTCGTCATGGGTGTGAAC \\
& R: ATGGCATGGACTGTGGTCAT \\
H-bFGF & F: AGAAGAGCGACCCTCACATCA \\
& R: CGGTTAGCACACACTCCTTG \\
H-TGF- $\beta 1$ & F: GGCCAGATCCTGTCCAAGC \\
& R: GTGGGTTCCACCATTAGCAC \\
H- $\alpha$-SMA & F: AAAAGACAGCTACGTGGGTGA \\
& R: GCCATGTTCTATCGGGTACTTC \\
H-Collagen I & F: GAGGGCCAAGACGAAGACATC \\
& R: CAGATCACGTCATCGCACAAC \\
H-Collagen III & F: GGAGCTGGCTACTTCTCGC \\
& R: GGGAACATCCTCCTTCAACAG
\end{tabular}

$\mathrm{H}$, human; bFGF, basic fibroblast growth factor; $\alpha$-SMA, $\alpha$-smooth muscle actin; $F$, forward; $R$, reverse.

transferred nitrocellulose membranes. Following blocking with 5\% skimmed milk for $1 \mathrm{~h}$ at RT, the membranes were incubated overnight at $4^{\circ} \mathrm{C}$ with the following primary rabbit antibodies: Anti-bFGF (1:1,000; cat. no. F3393; Sigma-Aldrich; Merck KGaA), anti-TGF- $\beta 1(1: 1,000$; cat. no. 3711; Cell Signaling Technology, Inc.), anti- $\alpha$-SMA $(1: 1,000$; cat. no. 19245; Cell Signaling Technology, Inc.), anti-Collagen I (1:1,000; cat. no. ab255809; Abcam), anti-Collagen III (1:1,000; cat. no. ab7778; Abcam) and anti-GAPDH (1:1,000; cat. no. 5174; Cell Signaling Technology, Inc.). The membranes were then incubated with a horseradish peroxidase-labeled goat anti-rabbit antibody (1:2,000; cat. no. SA00001-2; ProteinTech Group, Inc.) at RT for $2 \mathrm{~h}$. The protein bands were visualized with a chemiluminescent development agent $\left(\right.$ Chemistar $^{\mathrm{TM}}$ High-sig ECL Western Blotting Substrate; Tanon Science \& Technology Co., Ltd.). Protein expression was semi-quantified using GAPDH as the loading control by Image J software (2.0; National Institutes of Health).

Immunohistochemistry (IHC). The paraffin-embedded sections were prepared according to the protocol described in the 'Masson and H\&E staining' section. After deparaffinization and hydration, the paraffin-embedded sections were placed in a microwave for antigen retrieval at $120^{\circ} \mathrm{C}$ for $20 \mathrm{~min}$ in an autoclave, followed by washing with xylene and gradual rehydration in graded ethanol. Then, the sections were blocked with $3 \% \mathrm{H}_{2} \mathrm{O}_{2}$ at RT for $15 \mathrm{~min}$, and then further blocked with PBS containing 3\% BSA (cat. no. ST023; Beyotime Institute of Biotechnology) at RT for $30 \mathrm{~min}$. Next, the sections were incubated overnight at $4^{\circ} \mathrm{C}$ with the following rabbit antibodies: Anti-rat $\alpha$-SMA (1:150; cat. no. 41550; Signalway Antibody LLC), anti-rat Collagen I (1:200; cat. no. ab270993; Abcam) and anti-rat Collagen III (1:100; cat. no. ab7778; Abcam). Subsequently, the sections were incubated with a horseradish peroxidase-labeled goat anti-rabbit secondary antibody (1:4,000; cat. no. ab205718; Abcam) for $1 \mathrm{~h}$ at RT. The sections were developed using a DAB reagent kit (cat. no. DAB-1031;
Fuzhou Maixin Biotech Co., Ltd.) for $15 \mathrm{~min}$, and then counterstained with hematoxylin for 4 min at RT. After three washes with PBS, the sections were mounted onto slides with neutral gum and then observed under a CX43 light microscope (Olympus Corporation). Brown particles were regarded as positive staining. The number of positive cells per high power field was counted using Image $\mathbf{J}$ software (2.0; National Institutes of Health).

Statistical analysis. All statistical analyses were performed using GraphPad Prism software (version 9.00; GraphPad Software, Inc.). All experiments were repeated 3 times and data are presented as the mean \pm standard deviation. Comparisons among multiple groups were analyzed using one-way ANOVA followed by Tukey's post hoc test. $\mathrm{P}<0.05$ was considered to indicate a statistically significant difference.

\section{Results}

Impaired bladder function in rats receiving spinal cord transection is improved by telmisartan. After spinal cord transection, the cystometry test results were altered; rats in the model group displayed significantly higher bladder compliance, increased maximum cystometric capacity, increased residual urine volume, increased bladder wet weight and decreased detrusor pressure compared with that in the sham group (Fig. 1). After treatment with telmisartan, the values of the aforementioned parameters were significantly recovered, and the degree of recovery increased in a dose-dependent manner.

Disrupted bladder structure in rats receiving spinal cord transection is restored by telmisartan. The antifibrotic effect of telmisartan was evaluated b(1)y comparing changes in the content of collagen fibers and smooth muscle fibers in the bladder wall as determined by Masson and H\&E staining. The Masson staining results demonstrated that the bladder walls of rats that received spinal cord transection had disordered fibrous connective tissue, a distorted layered structure, increased thickness, reduced lamina propria, smooth muscle hypertrophy and increased numbers of intermuscular fibers compared with the sham group (Fig. 2A). Moreover, the H\&E staining results showed that the bladder detrusor cells in the sham group had a long spindle shape and were uniformly distributed, structurally tight and arranged in parallel (Fig. 2B). Compared with the sham group, rats that received spinal cord transection showed thickened bladder propria, hypertrophic and disordered detrusor cells, decreased numbers of muscle cells and increased amounts of intermuscular connective tissue. Telmisartan treatment relieved bladder tissue fibrosis and detrusor cell hypertrophy in rats that received spinal cord transection.

Increased fibrosis-related gene expression in rats receiving spinal cord transection is rescued by telmisartan. Western blotting and RT-qPCR were used to investigate changes in fibrosis-related gene expression. The results showed significantly increased expression levels of bFGF, TGF- $\beta 1$, Collagen I, Collagen III and $\alpha$-SMA expression in the smooth muscle cells of rat bladder tissue after spinal cord transection compared with those in the sham group (Fig. 3). Furthermore, telmisartan reversed the effects of spinal cord transection on 
A

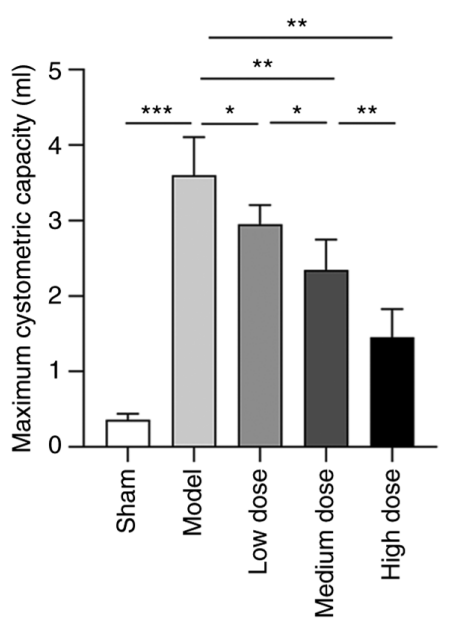

B

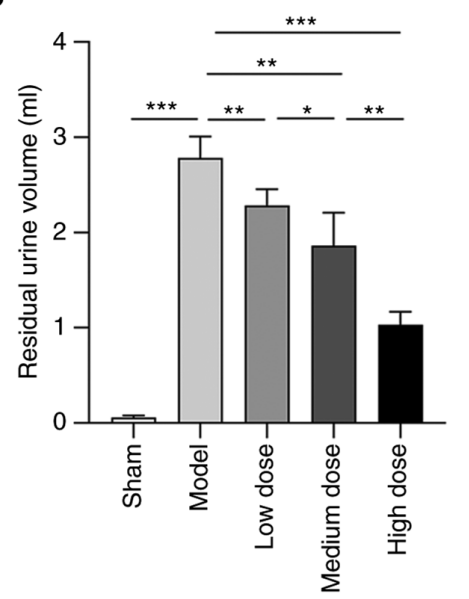

C

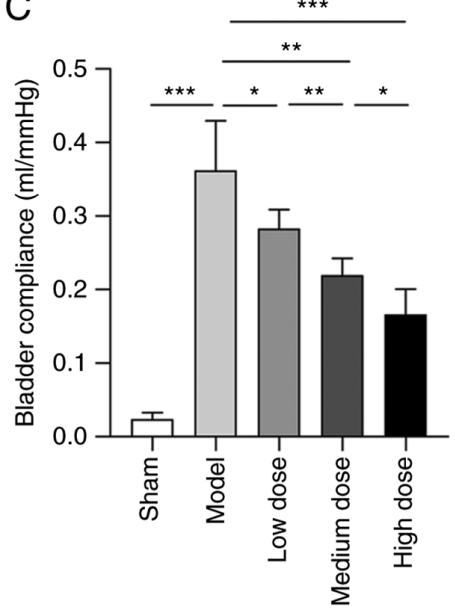

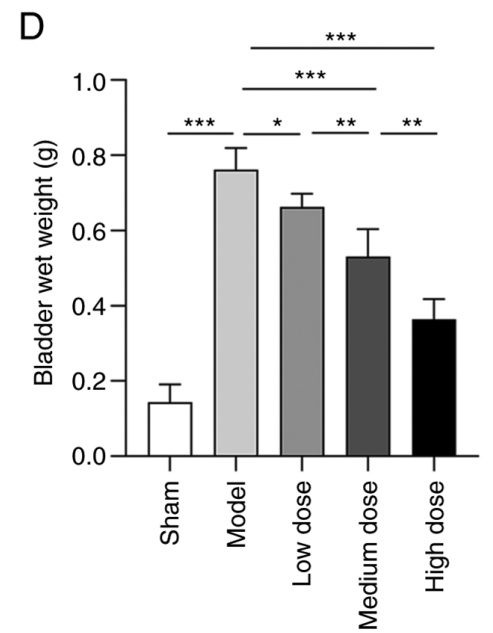

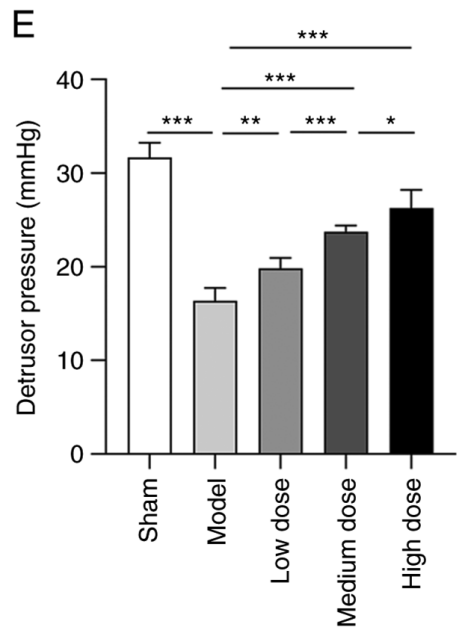

Figure 1. Cystometric analysis of the rats in each group. (A) Maximum cystometric capacity. (B) Residual urine volume. (C) Bladder compliance. (D) Bladder wet weight. (E) Detrusor pressure. ${ }^{*} \mathrm{P}<0.05,{ }^{* *} \mathrm{P}<0.01$ and ${ }^{* * * *} \mathrm{P}<0.001$.

A
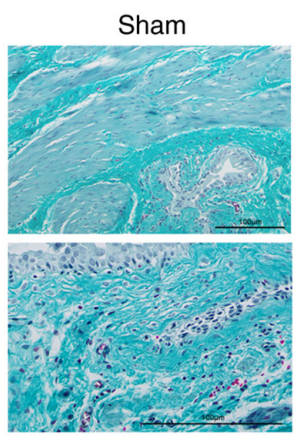

B

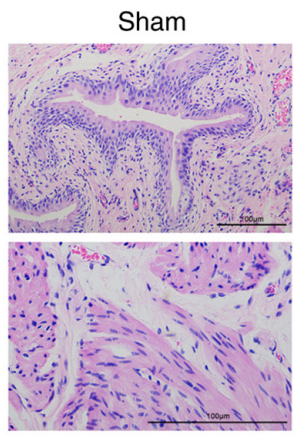

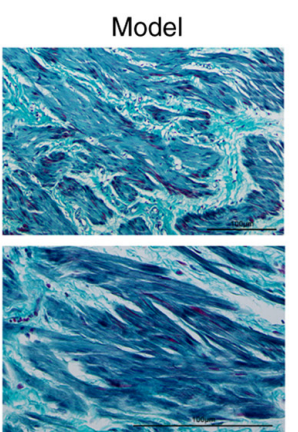

Model

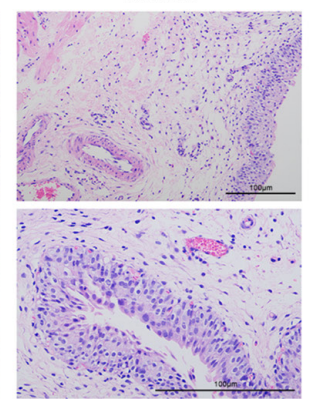

Low dose

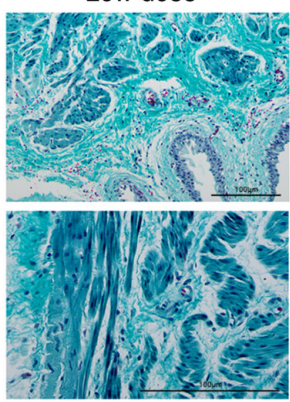

Low dose

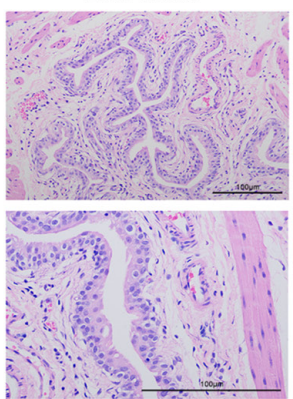

Medium dose
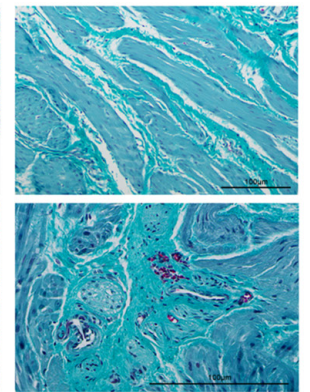

Medium dose

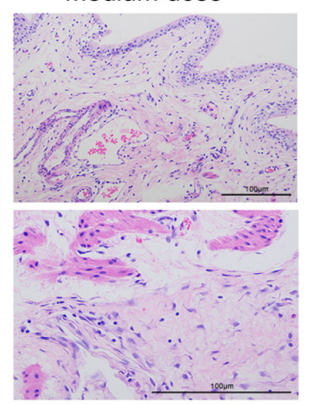

High dose

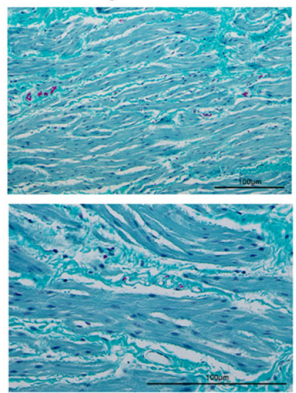

High dose

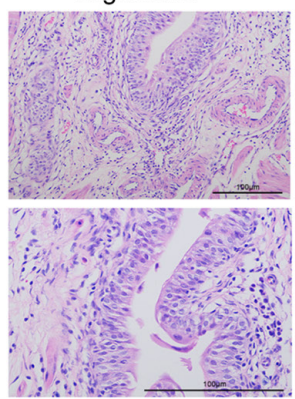

Figure 2. Morphological analysis of bladder tissue from the rats in each group. (A) Masson and (B) H\&E staining of bladder tissue from the rats in each group. Magnification, x200 and x400. 
A
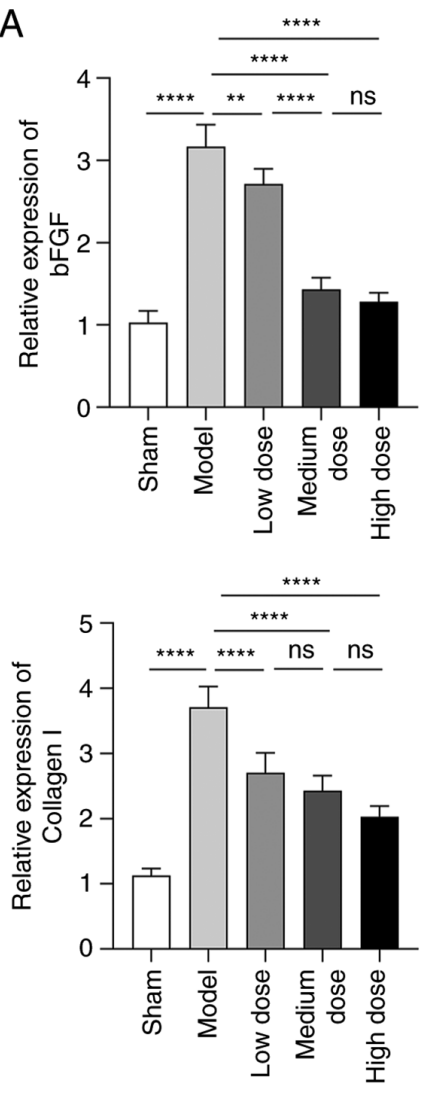

B
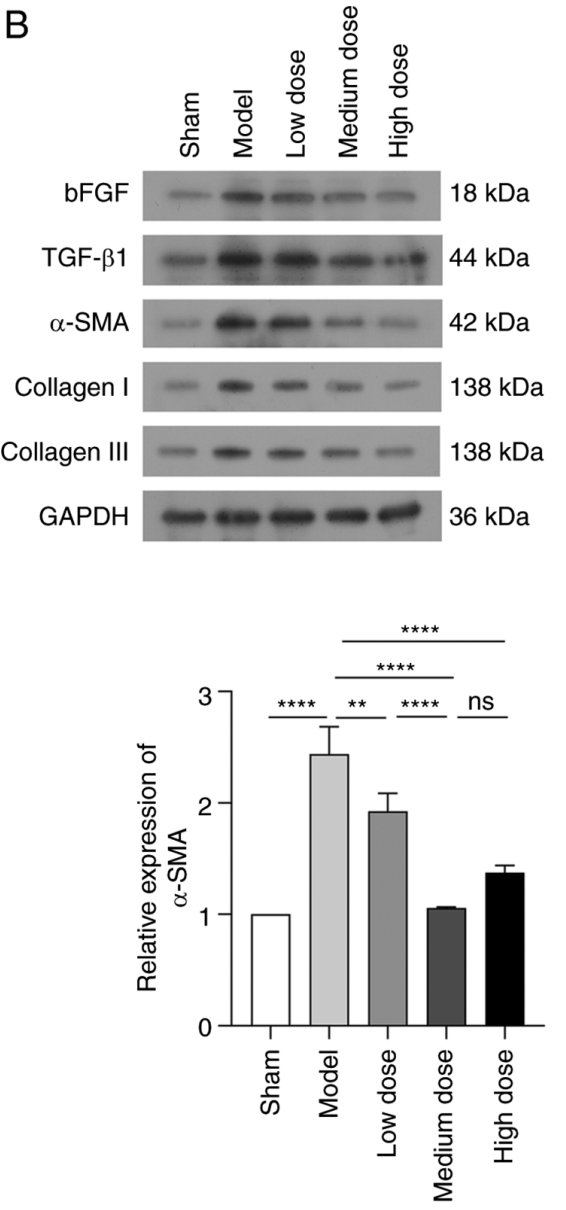
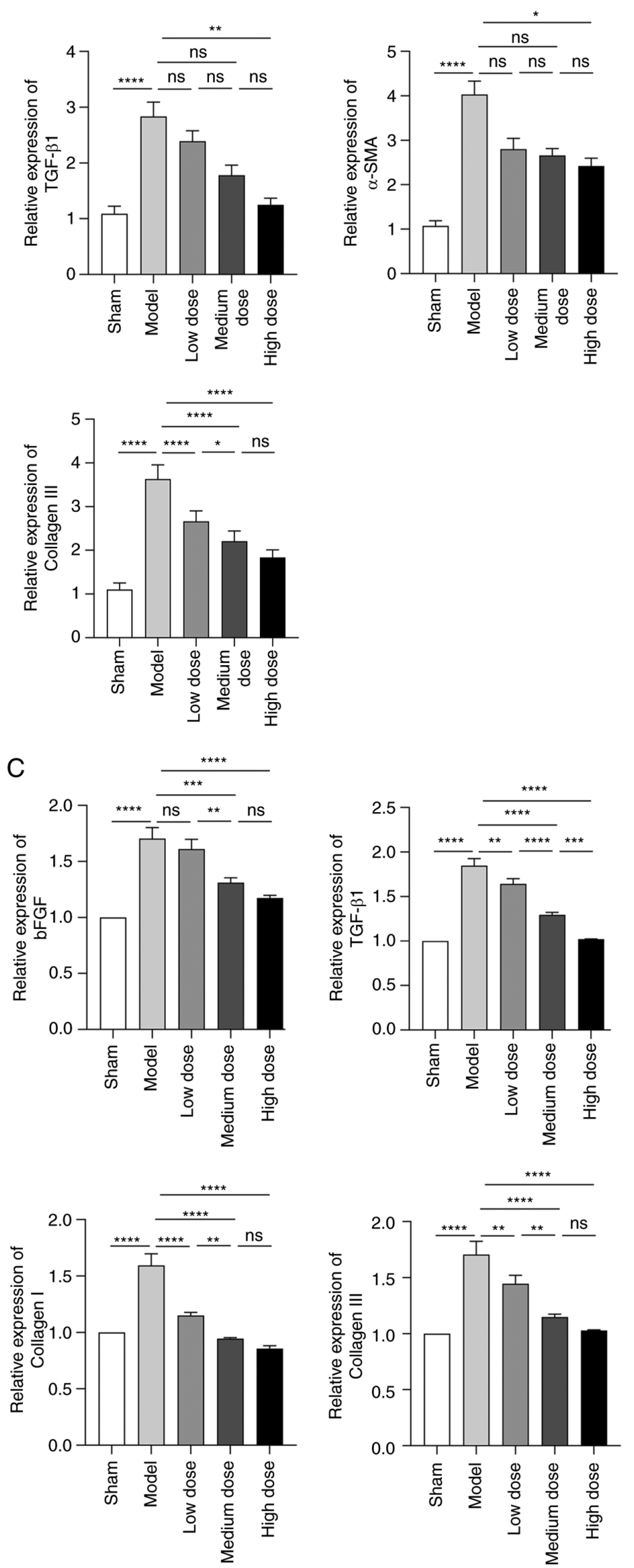

Figure 3. bFGF, TGF- $\beta, \alpha$-SMA, Collagen I, and Collagen III mRNA and protein expression levels in each group of rats. (A) Reverse transcription-quantitative PCR detection of bFGF, TGF- $\beta, \alpha$-SMA, Collagen I and Collagen III mRNA expression levels. bFGF, TGF- $\beta$, $\alpha$-SMA, Collagen I and Collagen III protein expression levels were (B) determined by western blotting and (C) semi-quantified. ${ }^{*} \mathrm{P}<0.05,{ }^{* *} \mathrm{P}<0.01,{ }^{* * *} \mathrm{P}<0.001$ and ${ }^{* * * * *} \mathrm{P}<0.0001$. bFGF, basic fibroblast growth factor; $\alpha$-SMA, $\alpha$-smooth muscle actin; ns, not significant. 
A
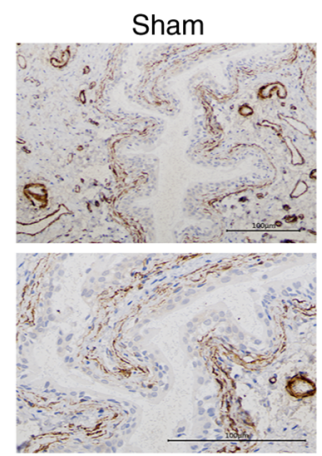

B
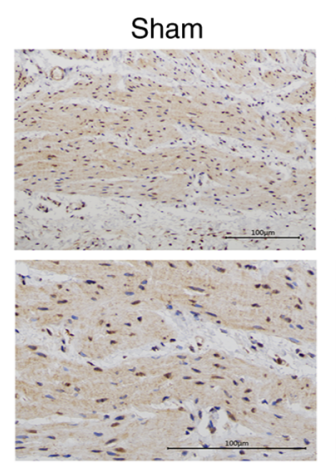

C
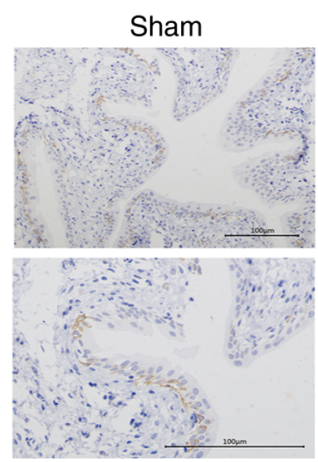

D

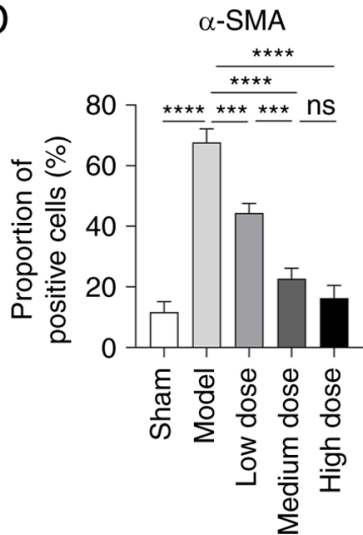

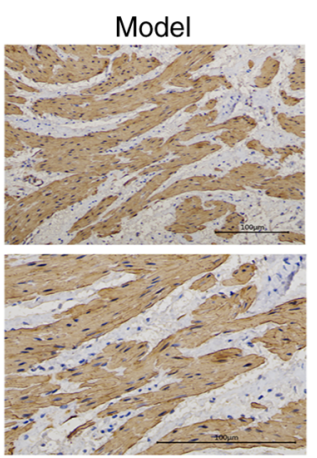

Model
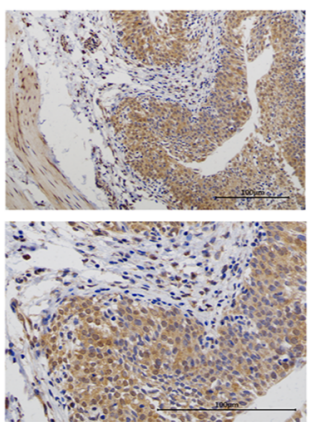

Model
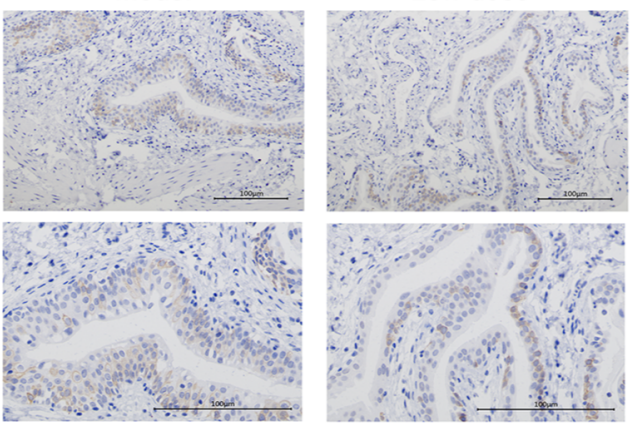

Collagen I

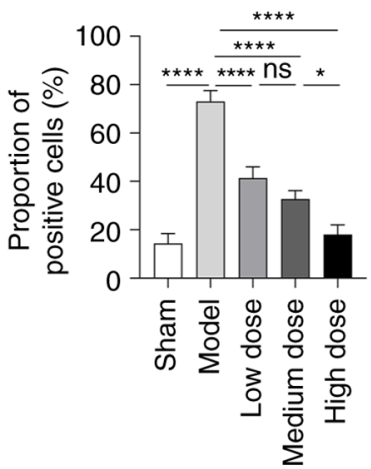

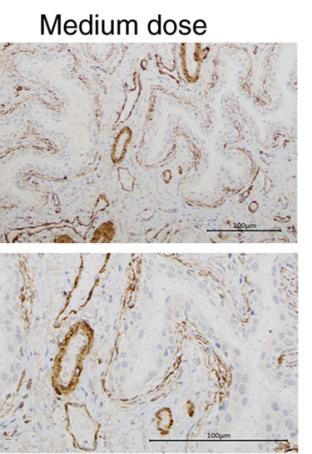
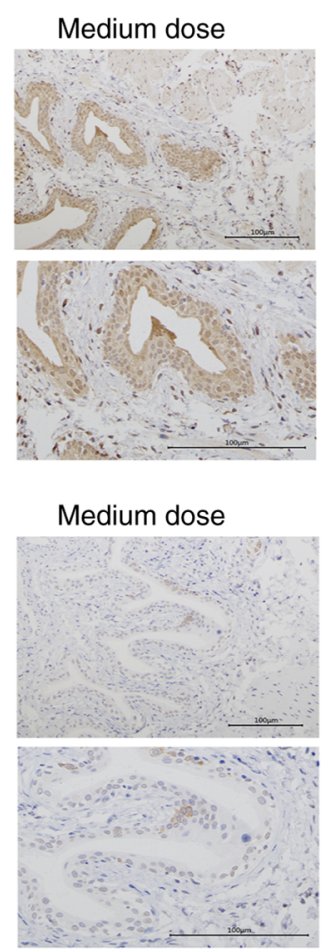

High dose

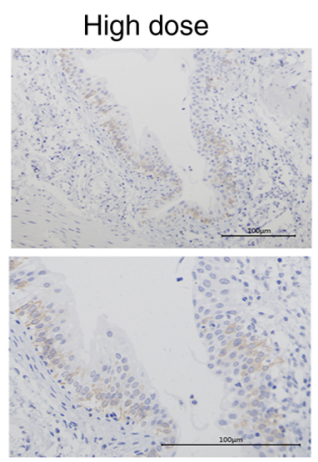

Collagen III
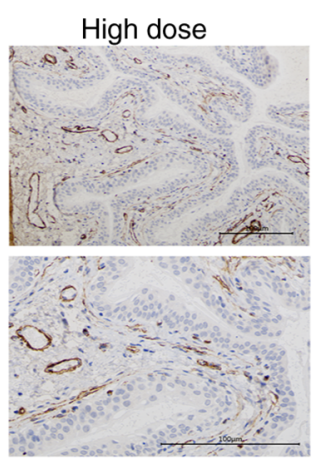

High dose
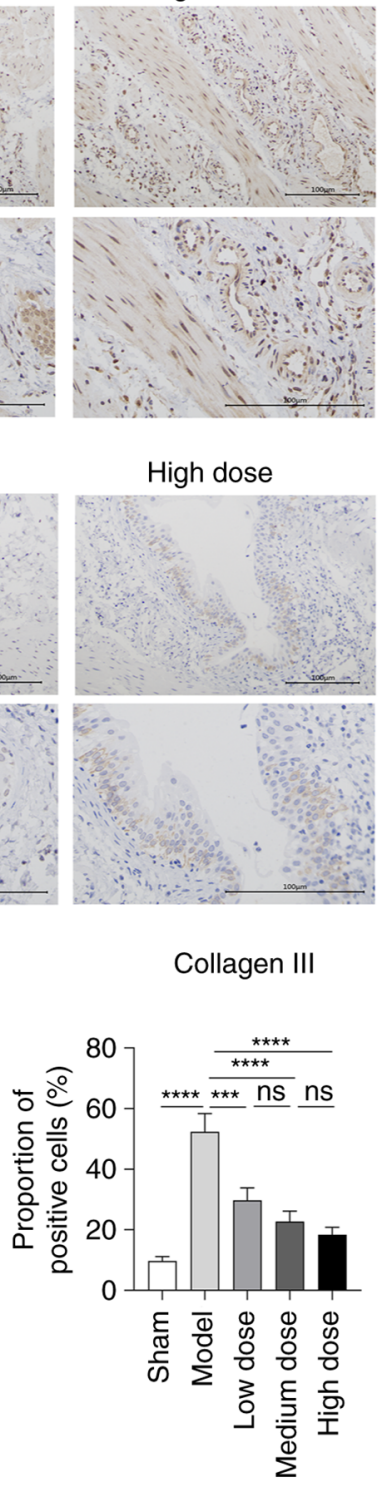

Figure 4. IHC staining of fibrosis-related proteins in the rat bladder tissues in each group. IHC staining of (A) $\alpha-S M A$, (B) Collagen I and (C) Collagen III. (D) Relative quantification of the expression of $\alpha$-SMA, Collagen I and III proteins. Magnification, $\mathrm{x} 200$ and $\mathrm{x} 400$. $^{*} \mathrm{P}<0.05,{ }^{* * *} \mathrm{P}<0.001$ and ${ }^{* * * * *} \mathrm{P}<0.0001$. IHC, immunohistochemistry; $\alpha$-SMA, $\alpha$-smooth muscle actin; ns, not significant.

the expression of fiber-related genes to various degrees. The IHC results showed the expression of target proteins in tissue in situ. The results demonstrated that the expression of Collagen I, Collagen III and $\alpha$-SMA was significantly increased after spinal cord transection compared with that in the sham group, whereas telmisartan treatment limited these effects (Fig. 4).

\section{Discussion}

An impairment of normal nervous system function that occurs due to an injury can be difficult to recover and affects the function of target organs, such as the bladder detrusor muscle (1). Although there is a link between hypertension and abnormal 
bladder function (21), to the best of our knowledge, the effect of ARB drugs on NB after an SCI has not been reported. The present study showed that treatment with telmisartan significantly reduced maximum cystometric capacity, residual urine volume, bladder wet weight and bladder compliance, and increased detrusor pressure in NB model rats. Telmisartan treatment also inhibited bladder tissue fibrosis and decreased the expression levels of bFGF, TGF- $\beta 1$, Collagen I, Collagen III and $\alpha$-SMA. Therefore, the present study provided supporting evidence for the use of telmisartan in treating NB after an SCI.

Current reports concerning changes that occur in bladder compliance after an SCI are inconsistent (22-24). A previous study showed that the bladder compliance of rats increased at 4 weeks after an SCI, but later decreased at 8 weeks after the SCI (25). This suggested that with a prolonged injury time, bladder compliance initially increases and then decreases. A potential explanation might be that in the early stage of injury, the bladder detrusor muscle had a low degree of fibrosis and only a small amount of collagen deposition. This allows for a compensatory enlargement of the bladder, which increases bladder capacity, lowers detrusor pressure and increases bladder compliance. Due to the long injury time, the bladder detrusor muscle may have become hypertrophic, fibrotic and degenerated, leading to a high degree of detrusor fibrosis, increased numbers of collagen fibers and decreased numbers of elastic fibers. These changes may result in a significant increase in detrusor muscle pressure and a gradual decrease in bladder compliance, which could provide an explanation for the results obtained in the present study.

It is generally believed that bladder wall fibrosis plays an important role in NB (26). In the present study, the Masson and H\&E staining results showed thickening of the bladder wall, disordered and hypertrophic detrusor cells, and proliferative collagen fibers in NB model rats. However, the short duration of the SCIs in the present study allowed for compensatory increases in maximum cystometric capacity and decreases in detrusor pressure. The collagen fibers in the bladder wall are primarily type 1 and 3 , and type 3 collagen is the determinant of bladder compliance (27). The present study indicated that the expression levels of Collagen I and Collagen III in the bladder detrusor muscles in the model group were not significantly increased, thus bladder compliance did not decrease. In addition, the protein expression levels of bFGF and TGF- $\beta 1$ in the model group were significantly higher compared with those in the sham group, indicating that the TGF- $\beta 1$ signaling pathway was activated in the NB model rats, leading to bladder fibrosis. Previous studies have shown that angiotensin II can promote cardiac fibrosis by binding to AngII type I receptors and further promoting the synthesis of TGF- $\beta 1$ (28-30). Telmisartan is a specific angiotensin II receptor antagonist. We hypothesized that telmisartan might have antifibrotic effects, which was confirmed by our experimental results. Therefore, the effect of the angiotensin II pathway on the TGF- $\beta$ pathway requires further investigation.

The Masson staining results showed that the bladder smooth muscles of rats that received spinal cord transection were hypertrophic and thickened, and displayed increased numbers of collagen fibers. We speculated that smooth muscle hypertrophy and thickening might be related to increased pressure in the bladder. High pressure is a harmful stimulus to smooth muscle cells, and may activate certain protein kinases in the cells and initiate abnormal cell proliferation $(31,32)$. This change is similar to that observed during liver fibrosis, as a previous study showed that pressure activates protein kinases, regulates gene transcription and initiates cell proliferation (33).

The present study had a number of limitations. Firstly, the experiments were conducted at 14 days after an SCI, which is at an early stage. Secondly, only the effects of three selected telmisartan doses were assessed, thus the dose-response relationship for further doses of telmisartan should be investigated in future studies. Thirdly, NB has several subtypes, meaning the results of the present study should be verified in further NB subtypes. Therefore, it is not clear whether the results of the present study can be directly applied to human NB. Finally, additional human bladder tissue samples should be analyzed to confirm the present findings. However, the present study suggested that potential strategies for preventing bladder fibrosis should be implemented as soon as possible for the treatment of NB, and telmisartan may serve as a useful therapeutic drug.

\section{Acknowledgements}

Not applicable.

\section{Funding}

This study was funded by the Jinan Science and Technology Development Plan (grant no. 201907074).

\section{Availability of data and materials}

The datasets used and/or analyzed during the current study are available from the corresponding author on reasonable request.

\section{Authors' contributions}

WC and QL conceived and designed the experiments. QL, RW and NM performed the experiments. $\mathrm{CW}$ analyzed the data. $\mathrm{QL}$ wrote the first draft. WC made the amendments and provided financial support. All authors read and approved the final manuscript. WC and QL confirm the authenticity of all the raw data.

\section{Ethics approval and consent to participate}

All experimental protocols were performed according to guidelines developed by the National Institutes of Health and were approved by the Institutional Animal Care and Use Committee of Hospital of Shandong University [approval no. KYLL-2021 (LW) 013].

\section{Patient consent for publication}

Not applicable.

\section{Competing interests}

All authors declare that they have no competing interests. 


\section{References}

1. Hamid R, Averbeck MA, Chiang H, Garcia A, Al Mousa RT, Oh SJ, Patel A, Plata M and Del Popolo G: Epidemiology and pathophysiology of neurogenic bladder after spinal cord injury. World J Urol 36: 1517-1527, 2018.

2. Przydacz M, Chlosta P and Corcos J: Recommendations for urological follow-up of patients with neurogenic bladder secondary to spinal cord injury. Int Urol Nephrol 50: 1005-1016, 2018.

3. Shang Z, Jia C, Yan H, Cui B, Wu J, Wang Q, Gao W, Cui X, $\mathrm{Li} \mathrm{J}$ and $\mathrm{Ou} \mathrm{T}$ : Injecting RNA interference lentiviruses targeting the muscarinic 3 receptor gene into the bladder wall inhibits neurogenic detrusor overactivity in rats with spinal cord injury. Neurourol Urodyn 38: 615-624, 2019.

4. Ge Q, Wang M, Lin Y, Xu C, Xiao J and Shen Z: Establishment of animal model manifested as bladder neurogenic changes generated by bilateral pelvic nerve injury in male rats. Int Urol Nephrol 53: 421-429, 2021.

5. Doyle C, Cristofaro V, Sack BS, Mahmood F, Sullivan MP and Adam RM: The role of the mucosa in modulation of evoked responses in the spinal cord injured rat bladder. Neurourol Urodyn 37: 1583-1593, 2018.

6. Soebadi MA, Bakula M, Hakim L, Puers R and De Ridder D: Wireless intravesical device for real-time bladder pressure measurement: Study of consecutive voiding in awake minipigs. PLoS One 14: e0225821, 2019.

7. Wyndaele JJ, Birch B, Borau A, Burks F, Castro-Diaz D, Chartier-Kastler E, Drake M, Ishizuka O, Minigawa T, Opisso E, et al: Surgical management of the neurogenic bladder after spinal cord injury. World J Urol 36: 1569-1576, 2018.

8. Romo PGB, Smith CP, Cox A, Averbeck MA, Dowling C, Beckford C, Manohar P, Duran S and Cameron AP: Non-surgical urologic management of neurogenic bladder after spinal cord injury. World J Urol 36: 1555-1568, 2018.

9. Li YL, Wen JJ, Wen YB, He XF, Wu JW, Li YW, Han ZJ, Feng JJ, Yan SH, Li SL, et al: Reconstruction of bladder function and prevention of renal deterioration by means of end-to-side neurorrhaphy in rats with neurogenic bladder. Neurourol Urodyn 37 1272-1280, 2018

10. Wada N, Shimizu T, Takai S, Shimizu N, Tyagi P, Kakizaki H and Yoshimura N: Combinational effects of muscarinic receptor inhibition and $\beta 3$-adrenoceptor stimulation on neurogenic bladder dysfunction in rats with spinal cord injury. Neurourol Urodyn 36: 1039-1045, 2017.

11. Duan LJ, Qi J, Kong XJ, Huang T, Qian XQ, Xu D, Liang JH and Kang J: MiR-133 modulates TGF- $\beta 1$-induced bladder smooth muscle cell hypertrophic and fibrotic response: Implication for a role of microRNA in bladder wall remodeling caused by bladder outlet obstruction. Cell Signal 27: 215-227, 2015.

12. Koeck I, Burkhard FC and Monastyrskaya K: Activation of common signaling pathways during remodeling of the heart and the bladder. Biochem Pharmacol 102: 7-19, 2016.

13. Sang R, Liu Y, Kong L, Qian L and Liu C: Effect of acellular amnion with increased TGF- $\beta$ and bFGF levels on the biological behavior of tenocytes. Front Bioeng Biotechnol 8: 446, 2020.

14. Ikeda Y, Zabbarova IV, Birder LA, Wipf P, Getchell SE, Tyagi P, Fry $\mathrm{CH}$, Drake MJ and Kanai AJ: Relaxin-2 therapy reverses radiation-induced fibrosis and restores bladder function in mice. Neurourol Urodyn 37: 2441-2451, 2018.

15. Cho KJ and Kim JC: Management of urinary incontinence with underactive bladder: A review. Int Neurourol J 24: 111-117, 2020.

16. Levanovich PE, Diokno A, Hasenau DL, Lajiness M, Pruchnic R and Chancellor MB: Intradetrusor injection of adult muscle-derived cells for the treatment of underactive bladder: Pilot study. Int Urol Nephrol 47: 465-467, 2015.
17. Drugs for hypertension. Med Lett Drugs Ther 62: 73-80, 2020.

18. Liu X, Shan X, Chen H, Li Z, Zhao P, Zhang C, Guo W, Xu M and Lu R: Stachydrine ameliorates cardiac fibrosis through inhibition of angiotensin II/transformation growth factor $\beta 1$ fibrogenic axis. Front Pharmacol 10: 538, 2019.

19. Chen Y, Ma Y, He Y, Xing D, Liu E, Yang X, Zhu W, Wang Q and Wen JG: The TGF- $\beta 1$ pathway is early involved in neurogenic bladder fibrosis of juvenile rats. Pediatr Res 90: 759-767, 2021.

20. Livak KJ and Schmittgen TD: Analysis of relative gene expression data using real-time quantitative PCR and the 2(-Delta Delta C(T)) Method. Methods 25: 402-408, 2001.

21. Torimoto K, Matsumoto Y, Gotoh D, Morizawa Y, Miyake M, Samma S, Tanaka N, Hirayama A and Fujimoto K: Overactive bladder induces transient hypertension. BMC Res Notes 11: 196, 2018.

22. Abolhasanpour N, Eidi A, Hajebrahimi S, Reyhani-Rad S and Hashim H: Effect of cerebrolysin on bladder function after spinal cord injury in female Wistar rats. Int J Urol 26: 917-923, 2019.

23. Salehi-Pourmehr H, Rahbarghazi R, Mahmoudi J, Roshangar L, Chapple CR, Hajebrahimi S, Abolhasanpour N and Azghani MR: Intra-bladder wall transplantation of bone marrow mesenchymal stem cells improved urinary bladder dysfunction following spinal cord injury. Life Sci 221: 20-28, 2019.

24. Lin CY, Sparks A and Lee YS: Improvement of lower urinary tract function by a selective serotonin $5-\mathrm{HT}_{1 \mathrm{~A}}$ receptor agonist, NLX-112, after chronic spinal cord injury. Exp Neurol 332: 113395,2020

25. Maciejewski CC, Tredget EE and Metcalfe PD: Urodynamic improvements following oral medical therapy for partial bladder outlet obstruction in an animal model. Neurourol Urodyn 34: 286-291, 2015.

26. He YL, Wen JG, Pu QS, Wen YB, Zhai RQ, Chen Y, Ma Y, Liu EP, Xing D, Ji FP, et al: Losartan prevents bladder fibrosis and protects renal function in rat with neurogenic paralysis bladder. Neurourol Urodyn 40: 137-146, 2021.

27. Hui J, Sharma S, Rajani S and Singh A: The specific molecular composition and structural arrangement of eleutherodactylus coqui gular skin tissue provide its high mechanical compliance. Int J Mol Sci 21: 5593, 2020.

28. Chen R, Feng Y, Wu J, Song Y, Li H, Shen Q, Li D, Zhang J, $\mathrm{Lu} \mathrm{Z,Xiao} \mathrm{H}$ and Zhang Y: Metformin attenuates angiotensin II-induced TGF $\beta 1$ expression by targeting hepatocyte nuclear factor-4- $\alpha$. Br J Pharmacol 175: 1217-1229, 2018

29. Wipff PJ, Rifkin DB, Meister JJ and Hinz B: Myofibroblast contraction activates latent TGF-beta1 from the extracellular matrix. J Cell Biol 179: 1311-1323, 2007.

30. Campbell SE and Katwa LC: Angiotensin II stimulated expression of transforming growth factor-beta1 in cardiac fibroblasts and myofibroblasts. J Mol Cell Cardiol 29: 1947-1958, 1997.

31. Thenappan T, Ormiston ML, Ryan JJ and Archer SL: Pulmonary arterial hypertension: Pathogenesis and clinical management. BMJ 360: j5492, 2018.

32. Frismantiene A, Philippova M, Erne P and Resink TJ: Smooth muscle cell-driven vascular diseases and molecular mechanisms of VSMC plasticity. Cell Signal 52: 48-64, 2018.

33. Zhang JG, Xing ZY, Zha TT, Tian XJ, Du YN, Chen J and Xing W: Longitudinal assessment of rabbit renal fibrosis induced by unilateral ureteral obstruction using two-dimensional susceptibility weighted imaging. J Magn Reson Imaging 47: 1572-1577, 2018 . 